\title{
ADOLESCENTES Y ADULTOS ¿ES POSIBLE UNA INTERACCIÓN SIN JUZGAR NI CASTIGAR? ¿QUÉ HAY DETRÁS DEL LLAMADO CONFLICTO GENERACIONAL?
}

\author{
Kattya Grosser Guillén ${ }^{1}$
}

Resumen: Me propongo con este ensayo describir a grandes rasgos las características del proceso adolescente, señalando cómo durante este momento que hemos dado en llamar adolescencia, se debe enfrentar el sujeto a una serie de transformaciones, una verdadera metamorfosis, que implica un posicionamiento distinto en la sociedad, la familia y la cultura en general. Es mi interés enfatizar en las implicaciones que tiene este proceso en las relaciones entre los y las adolescentes y los adultos, principalmente los padres y todo aquel adulto que ostente una posición de autoridad frente a ellos y ellas. Es mi interés ofrecer al lector una interpretación alternativa de lo que comúnmente se denomina el Conflicto Generacional, partiendo de la premisa de que si logramos como adultos comprender y respetar lo adolescente, será posible una interacción, si bien no exenta de conflicto, porque el mismo es inherente a lo humano y los vínculos que establecemos, menos violenta, más gratificante y provechosa para todos y todas.

Descriptores: ADOLESCENCIA, ADOLESCENCIA Y FAMILIA, CONFLICTO GENERACIONAL

\begin{abstract}
I set out with this Test in broad strokes to describe the characteristics of the adolescent process, indicating how during this moment which we have given in calling adolescence, the subject is due to face a series of transformations, a true metamorphosis, that implies a different positioning in the society, the family and the culture in general. It is my interest to emphasize in the implications that east process in the relations between the adolescents and the adults has, mainly the parents and all that adult whom a position of authority them shows in front of and they. It is my interest to offer to the reader an alternative interpretation of which commonly the Generational Conflict is denominated, starting off of the premise of which if we managed like adults to include/understand and to respect the adolescent, it will be possible interaction, although not free of conflict, because the same one is inherent to the human and the bonds who we establish, less does violence to, more rewarding and beneficial for all.
\end{abstract}

Key words: ADOLESCENCE, ADOLESCENCE AND FAMILY, GENERATIONAL CONFLICT

Si cuando se abre una flor al olor de la flor se le olvida la flor

Serrat

Es preciso aclarar que al proponerme escribir este ensayo acerca de las relaciones entre adultos y adolescentes, me es necesario referirme a lo adolescente, entendiendo con esto el proceso que transforma al niño en el sujeto adolescente y lo inscribe dentro de una estructura que llamamos adolescencia. Me orientan en este propósito además de los ejes teóricos psicoanalíticos, el reconocimiento de "los derechos adolescentes".

\footnotetext{
${ }^{1}$ Licenciada y Bachiller en Psicología de la Universidad de Costa

Rica. Profesora de las Escuelas de Psicología y Orientación y Educación Especial de la Universidad de Costa Rica. Atención clínica. Especialista en orientación psicoanalítica en adolescentes.

e-mail: kattiagrosser@hotmail.com
}

Artículo recibido: 28 de agosto, 2003

Anrobado: 24 de noviembre. 2003 
Precisamente por tener presente mi interés en reconocer y respetar los derechos de los y las adolescentes, he debido prestar especial cuidado en no caer en una visión adulto céntrica, entendida ésta como la posición sostenida desde el mito de la edad adulta bajo el supuesto de que la adultez es el momento final del desarrollo humano, donde hay un acabamiento y la consecución de una madurez y estabilidad emocional, según el cual han desaparecido las contradicciones y se tienen las certezas, supuesto momento donde junto a las preguntas están las respuestas.

Mito, que está destinado a su caída junto con su carga de valoración ideológica y al reconocimiento de que nunca se accede a un estado adulto ideal y perfecto, en el que culmina todo desarrollo previo. Ya que, conceptos como los de madurez, estabilidad, equilibrio y adultez han entrado en crisis, puesto que forman parte de las ideologías represivas, al servicio del mantenimiento del orden establecido. (Tuber, 1988, p 15)

\section{Lo adolescente}

¿Qué le va usted hacer, Señora. Si en su reloj sonó la hora De olvidar vuestro hogar, Señora

Serrat

Comparto la conceptualización del desarrollo humano como un proceso en espiral, donde el crecimiento, los conflictos y las dudas permanecen siempre y son el motor del desarrollo y el cambio. Posición teórica que en mi opinión corresponde a una visión menos pretenciosa de lo humano en general y del adulto en particular. Retomando a Tubert, creo con ella, que

Solo se puede concebir el proceso de desarrollo en términos de lucha de contrarios, tal como se pone en evidencia con toda claridad en la adolescencia, que nos permite observar particularmente las sucesivas síntesis de integraciones y desintegraciones de progresos y regresiones (Tuber, 1988, p. 13)

Aún cuando el conflicto, la duda y la contradicción están presentes en la vida adulta, me parece que la adolescencia es un momento privilegiado para la expresión de estos, y es el momento donde con mayor claridad se observan los continuos avances y retrocesos, siendo un momento que se reconoce, justamente a partir de las contradicciones, las aparentes regresiones y la fluctuación de sentimientos. 
Algunos autores han llamado y conceptualizado la adolescencia como la transición, otros el pasaje y algunos una fase antes de la edad adulta. Hay quienes incluso la definen como un momento en que ya no se es niño pero tampoco se es adulto, como aparece en algunos textos generales sobre el desarrollo adolescente: "La adolescencia es un período de transición durante el cual los miembros del grupo cambian desde la niñez hasta la edad adulta. El adolescente pertenece en parte al grupo de los niños y en parte al del adulto" (Rice, 1994, p. 41 El subrayado es mío ). Este tipo de definiciones tienden a inducir a error, arrebatándole a lo adolescente un lugar propio e invalidando con esto sus derechos.

La adolescencia es parte del proceso de estructuración sujetiva, y es una parte fundamental, igualmente importante que los primeros cinco años de vida, tan vital y determinante como éstos. Silvia Tubert plantea que en esta estructura llamada adolescencia "se rescribe todo lo construido hasta el momento de su cristalización, y a su vez, persistirá posteriormente, resignificándose de continuo de diferentes maneras, en función de las experiencias del sujeto y de sus relaciones con el universo simbólico del que forma parte." (Tubert, 1982, p. 16) Resignificar, es darle un lugar distinto, significarlas de una manera nueva, lo que es posible en la adolescencia, pues el sujeto cuenta con nuevos recursos intelectuales y emocionales. Ia salida del circuito familiar, como único referente, posibilita incursionar con nuevos vínculos y nuevas formas de vincular, las figuras de identificación no son ya, y a partir de ese momento no lo serán más, en exclusiva los vínculos primarios, esto permite al sujeto tener nuevos referentes y con ello construir-se desde un lugar distinto.

Después de este pasaje el sujeto es otro, dice Bercovich y continua "asistimos a un cambio de posición sujetiva... El sujeto atraviesa ciertos nudos en los que todo es puesto en cuestión. Puntos subjetivantes en tanto hacen a la estructura del sujeto, una vez atravesados, el sujeto deviene otro." (Bercovich,1994, p. 131) De tal forma que lo que ocurra durante este proceso cobra una importancia vital, es un proceso posibilitador en tanto hace posible ubicarse un lugar distinto al asignado en su familia de origen, posibilitador en tanto el sujeto podría desprenderse de depositaciones y designios infantiles, posibilitador en tanto las experiencias infantiles valorizantes o desvalorizantes, pueden resignificarse.

Durante este proceso la persona tiene un papel activo, no como a veces se desprende de algunos abordajes que sobre la adolescencia se realizan, donde pareciera que la persona es un ente pasivo al que le ocurren una serie de transformaciones emocionales producto del 
desarrollo sexual, principalmente. Visión de la adolescencia que a veces conduce al error de suponer que el niño y la niña al llegar a este momento de su desarrollo es atropellado (a) por un torbellino de emociones contradictorias y de exigencias sociales a las cuales debe responder y acomodarse, proceso que, no en pocas ocasiones, se espera con ansia que termine, pues se está convencido que al acabar esta "etapa", la persona alcanzará la "madurez" y logrará insertarse, sin incomodar en la sociedad. Subyace en estas concepciones una visión adultocéntrica y normalizante, pero más allá de esto, lo que me parece más grave, es que se le niega a la adolescencia el lugar preponderante en la constitución psíquica humana, prácticamente dejando este proceso casi que exclusivamente a la infancia.

Atravesar la adolescencia, es fundamental para toda persona. Pero ser adolescente no es únicamente alcanzar la madurez de los órganos sexuales y su consecuencia la adquisición de los caracteres sexuales secundarios, ni tampoco enfrentar las nuevas exigencias sociales, como por ejemplo plantearse un proyecto de vida, tener una pareja, adquirir una conciencia moral. Es mucho más profundo que eso, implica un proceso psíquico, Ricardo Rodulfo (1986) plantea que se trata de puntos de estructuración del sujeto que se llevan a cabo en la adolescencia y que él los conceptualiza como trabajos.

Emplea el concepto de trabajo como una labor que debe llevar a cabo el sujeto, que lo coloca en una posición activa y no como un ente pasivo al que las circunstancias atrapa, y entonces algo le sucede. Por el contrario se trata de un trabajo psíquico que le pertenece al sujeto, que implica su propia actividad para llevarlo a cabo, de acuerdo a los recursos de que dispone para realizarlo. $Y$ estos trabajos suponen una serie de actitudes que de no tenerse en cuenta como necesarias para la propia estructuración sujetiva, se puede caer en el error de querer evitar que se den, o incluso, como ocurre a menudo, podrían tomarse como síntomas patológicos.

Para hablar de un proceso adolescente, se requiere que se estén llevando a cabo estos trabajos simbólicos, que implican, una posición muy distinta del sujeto frente a su familia, frente a los adultos y figuras de autoridad, distinta en relación con el niño o la niña que fue.

Estos cambios, son en esencia lo adolescente, por lo general se producen en cierto período de edad, entre los 12 y los 19 años, la adolescencia, pero no necesariamente hacen su 
aparición únicamente en este momento, pueden presentarse en el transcurso de una vida, en diferentes momentos y frente a situaciones críticas, sin embargo cuando esto ocurre más allá de la adolescencia se tiene por lo general la autonomía que la vida adulta posibilita y por tanto no resultan tan amenazantes para los otros. En la adolescencia, lo adolescente suele resultar muy amenazante para la familia, los adultos en general y la sociedad en su conjunto. Tal vez por resultar tan amenazantes suelen combatirse y en muchos casos obstaculizárseles. En tal grado se desconoce lo fundamental de este proceso que se plantea como ideal, cuando no se observan los signos de él. No en pocos casos los y las adolescentes tienen que luchar con todas sus fuerzas para que se les permita ocupar un lugar distinto.

La adolescencia brinda la oportunidad de reparar o recomponer traumas infantiles, al tener la posibilidad de este desprendimiento familiar y contando con un afuera que funciona como un nuevo continente, implica que el sujeto está en condiciones de sobreponerse a vivencias negativas de la infancia, e incluso superarlas, desdibujando sus trazos, de tal forma que no conduzcan inevitablemente a la elaboración de síntomas o francas desestructuraciones.

El adolescente está atravesando un proceso de construcción de su ser, por eso los vemos ensañando posturas, y posiciones. Partiendo de sí mismo, de ese que empieza a descubrir que es y buscando figuras de identificación: personas, instituciones, ideologías, causas, palabras. Identificaciones que pueden ser determinantes para el desenlace de quién será. Lo que encuentre en el afuera puede marcar una diferencia en el rumbo que tome o al que se encamine el sujeto adolescente. Esto representa una oportunidad y un riesgo. Oportunidad en tanto puede reconectar su libido con personas y proyectos que le posibiliten un crecimiento y desarrollo de sus potencialidades o a la inversa podría correr el riesgo de reconectarse con figuras y proyectos negativos que impidan un desarrollo y canalización positiva de sus potencialidades. De allí lo determinante del medio en que el adolescente incursione.

En los últimos tiempos se ha dado una mayor atención a la adolescencia admitiendo que no se pueden incluir dentro de la categoría niñez y se empiezan a incluir en los discursos. Sin embargo esta inclusión es, en su mayoría, desde el lugar de "problema", resaltando las "conductas de riesgo" en algunos casos, la necesidad de "controlarlos" en otros, colocándolos en un lugar donde lo característico es la impulsividad, la falta de control, la 
desorientación y la irresponsabilidad. Vasta ver en los periódicos, revistas y programas televisivos donde se destina espacios al tema, para comprobar que los mismos se orientan a temas como "Embarazo en la adolescencia", "Adolescencia y pandillas o barras", "Adolescencia y consumo de licor, tabaco y drogas ilegales", "Adolescencia y deserción escolar". Me pregunto ¿la capacidad creativa? ¿la búsqueda de transformación? ¿la innovación? ¿su capacidad crítica? ¿su derecho a cuestionarnos, a los adultos y a la cultura en general? ¿su derecho a la expresión? ¿su derecho a un espacio propio y un lugar en la sociedad? Todo esto también hace parte de lo adolescente, reconocerlo y destacarlo es darle un lugar, que en mi opinión la sociedad en términos generales les niega.

Hasta antes del siglo XX y sobre todo durante el proceso de industrialización, los derechos de los niños no era reconocidos, y los niños entre otros fueron profundamente explotados y violentados. Creo que en la modernidad, en alguna medida, los y las adolescentes han sido los ignorados, a partir de la imposibilidad de otorgarles un lugar propio y un reconocimiento y aceptación de este. Lugar que la juventud en general ha conquistado.

Baste recordar la década de los sesenta: verdadera revolución de signos, expresión de ruptura con toda moral y valor. Años de fecunda creatividad que hicieron época y estilo dejando huellas psicodélicas en el arte, la música, la literatura y la historia. La cultura occidental de hoy, así como cada uno de nosotros, lleva la marca del movimiento en el cual la adolescencia tuvo un papel protagónico. (Bercovich, 1994, p. 136)

Sin embargo, después de la presencia que tuvo la juventud en los años 60, el protagonismo y beligerancia que demostraron, la historia oficial en gran medida, ha tendido a descalificar dicho protagonismo reduciéndolo a la "rebeldía sin causa", el desenfreno, las drogas y el intercambio sexual irresponsable.

En nuestro país desde 1998, se cuenta con el Código de la Niñez y la Adolescencia. Se nombran y al nombrarse se visualizan, los y las adolescentes; así el artículo 2 de dicho Código dice "Para los efectos de este Código se considerará niño o niña a toda persona desde su concepción hasta los doce años de edad cumplidos y adolescente a toda persona mayor de doce años y menor de dieciocho..." (Código de la Niñez y la adolescencia, 1998, p. 136). Dicho Código representa un avance en tanto brinda un lugar diferenciado a los y las adolescentes, reconociendo que aún cuando son menores de edad, no pueden ser incluidos 
en la categoría niñez, admitiendo que constituyen un segmento de la población, con características y necesidades propias.

Aún cuando hoy en día se habla de los derechos de los y las adolescentes, los adultos parecen no aceptarlos, por ignorancia o negación. No se puede respetar lo que no se reconoce, no se va a respetar el derecho a la expresión de los y las adolescentes si no se cree que tengan algo importante que decir; no se puede respetar el derecho a la libertad de opinión de los y las adolescentes, si se cree que no saben pensar, que son impulsivos y poco reflexivos. ¿Cómo respetar el derecho a la información de los y las adolescentes si se cree que el problema de la juventud actual es que no obedece a los mayores?.

¿Por qué es tan difícil, para los adultos, aceptar y respetar lo adolescente? ¿Por qué no ha sido difícil reconocer los derechos de los niños? ¿Por qué se aceptan y toleran con gusto y cariño las características infantiles, con todo lo agotador de la demanda infantil hacia los adultos, necesarios inevitables para su pleno desarrollo?

Me atrevo a aventurar una respuesta. Durante la infancia los adultos cercanos son los ideales, los infantes confían plenamente en ellos. Necesitan creer en los adultos, suponer que lo saben todo y que todo lo pueden. Los niños y las niñas desean aprender de ellos, están dispuestos y deseosos a aprender lo que los adultos deseen enseñarles. Tienen que confiar, para dejarse guiar, dado su alto grado de dependencia.

Por el contrario, los adolescentes hacen su entrada en lo social transgrediendo, cuestionando, buscando la verdad, que los adultos no tienen, criticando la sociedad en general. Para los y las adolescentes todos los adultos estamos cuestionados. Es por este cuestionamiento que los adolescentes resultan tan amenazantes para los adultos y quizá por eso mismo es tan difícil aceptarlos.

Para cualquier adulto posicionarse como tal frente a un o una adolescente implica estar en posición de ser cuestionado, estar expuesto a su crítica aguda, a su duda y desconfianza.

Para el sujeto adolescente, este cuestionamiento y puesta en duda es necesaria, finalmente partiendo de éste, podrá construir su propia línea de pensamiento, creencias y valores. Si no lo hace no habrá para ellos una verdadera elección personal, sería pura repetición, sumisión 
y/u obediencia con respecto a la posición de sus padres, madres y otros adultos a los cuales los y las adolescentes imiten. Esta sumisión no permitiría al adolescente desarrollarse como sujeto independiente, no le permitiría ostentar un criterio personal, y lo dejaría en desventaja para enfrentarse a la toma de decisiones, allí donde no estén los padres ni otros adultos para decidir por él o ella.

Poder interpretar este permanente cuestionamiento como un proceso necesario y no simple altanería, es indispensable para que la relación con lo adolescente no sea, únicamente, un enfrentamiento poco productivo.

\section{La familia}

Yo soy ese por quien ahora, os preguntáis por qué, señora se marchitó vuestra fragancia, perdiendo la vida, mimando su infancia, velando su sueño, llorando su llanto con tanta abundancia

Serrat

Para el sujeto adolescente la familia deja de ser el centro de donde partir para mirar el mundo y pasa a ser el referente, para comparar y cuestionar. Mirando no ya desde adentro, sino, como si estuviera afuera, colocándose en el lugar de juez y verdugo.

El trabajo psíquico que el sujeto adolescente debe llevar a cabo implica resignificar sus experiencias infantiles, esto implica, religar, desligar y volver a ligar muchas de estas experiencias y vivencias infantiles, darles una nueva significación y esto lo lleva a posicionarse en un lugar distinto.

Este proceso no es lineal, y como todo en lo humano es contradictorio, e implica grandes conflictos y fluctuaciones de sentimientos. El sujeto adolescente se muestra por tanto muy cambiante, hay momentos en que se retrae y vuelca al seno familiar con la actitud infantil y dependiente, en busca de contención y la seguridad perdida, por el contrario hay momentos en que con fuerza y a veces con severidad manifiesta su oposición a ésta y su deseo de estar fuera del área de influencia de sus padres a quienes vive como amenazantes en tanto siente que la familia lo devuelve al mundo infantil y le impide su crecimiento. En la medida 
de que la familia manifieste su deseo de reintegrarlo desde el mismo lugar infantil, más violento puede ser el desprendimiento para el o la adolescente y para sus padres.

El sujeto adolescente, al descubrir que, su mundo infantil en mucho estaba fundado en una idealización, se siente defraudado, y al constatar que su familia no es la ideal, culpa a los adultos de esta constatación y se siente engañado.

No están en juego los afectos, el o la adolescente no deja de querer a sus padres o a su familia, lo que ocurre es que ésta ocupa un lugar distinto. Proceso que Ricardo Rodulfo (1989) llama el pasaje de lo familiar a lo extrafamiliar y lo describe como "una metamorfosis", una transformación...por primera vez lo extrafamiliar deviene más importante que lo familiar. Si bien es cierto lo extrafamiliar no aparece con la adolescencia, es la primera vez que lo extrafamiliar pone en duda y cuestionamiento lo familiar, la preeminencia de lo familiar en la primera infancia, por ejemplo, es lo que varía.

Durante lo que conocemos como período de latencia, hay obviamente todo un trámite de lo extrafamiliar que se va desarrollando y que, se espera que lo extrafamiliar tenga mucho peso, por ejemplo a través de la escuela y los amigos. Tampoco acá, aún con esto, no basta para quebrantarle a lo familiar su primacía. Este es un hecho nuevo que solo acaece después de la pubertad, y que no le viene regalado al adolescente, lo tiene que conquistar. (Rodulfo, 1989, p. 156)

Los padres por otra parte están viviendo su propia crisis, pues han sido destronados y esto representa para ellos una herida narcisista no fácil de soportar. Pasar de ser quienes tenían el saber y el poder casi absoluto, para pasar a ser los cuestionados y puestos a prueba en todo momento, reconocer que ya no están seguros de su papel, reconocer no solo que los hijos los superan en fuerza y tamaño sino también en vitalidad y energía, implica para los padres una contienda de grandes proporciones consigo mismos y con sus hijos (as). Hace reaparecer su propia adolescencia, sus conflictos edípicos y sus sueños truncados. Les recuerda el paso del tiempo y los confronta con sus proyectos y el tiempo que les queda.

Los adultos, también atraviesan un enfrentamiento con la muerte, con las pérdidas y también es preciso que realicen sus propios duelos. El paso del tiempo es una vivencia totalmente distinta para un adulto, que para un adolescente. El tiempo es una dimensión absolutamente subjetiva, para un adulto después de haber vivido 35 o más años, se puede decir con Gardel 
que "veinte años no es nada". Para un o una adolescente es inimaginable, para ellos y ellas, veinte años son demasiados, es como una hoja en blanco donde todo es posible. Todo es posible no solo por esta particular manera de dimensionar el tiempo sino también por su omnipotencia.

La omnipotencia es un mecanismo necesario e inevitable del proceso adolescente, responde a la regresión narcisista por la que atraviesa, es claro que los y las adolescentes no lo pueden todo, pero así lo creen. Como adultos no se trata de demostrárselo, como se intenta a veces con violencia e irrespeto. Los y las adolescentes tendrán que descubrirlo y los adultos pueden y deben colaborar en ese descubrimiento. Quizá los y las adolescentes puedan más de lo que los adultos suponen y menos de lo que ellos y ellas esperaban.

Durante la adolescencia, las certezas que venían de la familia y los adultos cercanos, que hacían sentirse seguro en la infancia, se transforman en dudas. Al descubrir que ya no hay certezas, que no existe una verdad, y si existe será una particular para cada individuo y que la suya está por construirse, se siente como un equilibrista, sobre una cuerda floja y sin entrenamiento. Para defenderse de esta sensación los y las adolescentes tienen la omnipotencia, entonces sienten que todo lo pueden, que basta con imaginar para transformar la realidad. Esto les da la potencia y la arrogancia que los caracteriza y que los adultos rechazan, más por temor y desconocimiento que por simple intolerancia.

Los adultos tenemos la obligación y la responsabilidad de orientar y marcar los límites de lo posible de acuerdo a la realidad, en algunos casos los y las adolescentes requieren experimentar y en muchos podrán tener éxito, aún cuando no en todo, por supuesto, sin embargo, es su derecho descubrirlo por sí mismos y obtener sus propios aprendizajes para poder extraer sus propias conclusiones.

\section{La cultura}

Póngase usted un vestido viejo Y, de reojo en el espejo, Haga marcha atrás, Señora Recuerde antes de maldecirme, Que tuvo usted la carne firme $Y$ un sueño en la piel

Serrat 
La humanidad es hoy lo que es producto de la innovación que las nuevas generaciones aportan a las anteriores. De no ser por la terquedad y el ímpetu de los que se han atrevido a cuestionar lo que se pensaba como incuestionable, ni la ciencia ni la medicina, ni la tecnología y en general el conocimiento humano sería hoy lo que es.

Para el adolescente su grupo, sus amigos, su novio o novia, compañeros y compañeras, sus pares, cómplices de aventuras, fantasías y proyectos son las personas de mayor importancia, en ellos confían y creen. Con ellos aprenden desde mirarse en el otro como en un espejo, hasta de su confrontación y diferenciación. El grupo de iguales es indispensable para el proceso de constitución de su identidad.

Los y las adolescentes van a defender a su grupo con fuerza y empeño, es completamente inútil insistir en desmitificar o desacreditar a sus pares. El adolescente lo vivirá como una agresión y responderá de la misma manera, o de la manera más común en que se puede reaccionar frente a una agresión, defendiéndose.

La adolescencia, los y las adolescentes producen una profunda herida narcisista en los adultos, con su proceso y su presencia obligan a reconocer a los adultos que tampoco se tiene la verdad, que no es cierto que se tenga todas las respuestas y que en muchos casos ni siquiera se tienen las preguntas. Reaccionar sin violencia, soportar esta herida sin ira es el reto para las y los adultos.

Para el adulto es una tarea difícil tolerar el cuestionamiento y la crítica a veces despiadada de los y las adolescentes y frente a esto puede aflorar la tentación, de parte del adulto, de enfrentarse al joven, desde su lugar de poder y hacer uso y abuso de éste para recordarle al joven su lugar dependiente, intentar someterlo por la fuerza y su posición vulnerable en la sociedad. Es una respuesta violenta del adulto, urgido de recuperar su poder puesto en cuestión por los adolescentes. Es cuando se esbozan ideas descalificantes para lo adolescente, es cuando la raíz de la palabra se hace arma: Adolescente que adolece, se enfatiza a conveniencia este adolecer, se describen peyorativamente algunas de las características de la adolescencia, se intenta resaltar la inexperiencia, colocando la experiencia como la única fuente válida de saber, se descalifica el idealismo adolescente como pensamiento irracional y fantasioso. Se recuerda con arrogancia que los adultos transitaron estos caminos mucho antes de que ellos nacieran y con rabia abiertamente 
expresada o solapadamente se les dicta, cual premonición, que esos ideales que ahora sostienen con vehemencia, necesitarán unos pocos años para ser olvidados por imposibles.

Posicionado el adulto en ese lugar descrito, es cuando las relaciones entre generaciones se vuelve una guerra. Los y las adolescentes les recuerdan a los adultos su propia adolescencia y con ella todos los sueños que quedaron truncados, olvidados o a los cuales se tuvo que renunciar por las dificultades que la vida impuso como prioridades; recuerda el paso del tiempo y la juventud pasada; surge la preocupación por el tiempo que empieza a parecer poco en relación con los proyectos. A veces los adultos quieren transferir esa experiencia a los adolescentes, hacerles entender que el tiempo pasa más rápido de lo que ellos se imaginan en este momento y que las oportunidades se pueden esfumar si no se aprovechan, que los sueños se logran con mucho más esfuerzo que imaginación, que la imaginación es importante pero insuficiente. Todo esto podría ser verdad, pero es la verdad de los adultos, no es la verdad del adolescente y no tiene por qué serlo. El encuentro de estas dos verdades suscita un enfrentamiento que es desgarrador pero no tiene por que ser violento ni destructivo.

La posición adolescente es tan válida y tan real como la del adulto, una no anula la otra. Es posible que este encuentro de generaciones sea constructivo y disfrutable para todos, si los adultos permiten y reconocen el derecho de los y las adolescentes de atravesar este proceso y aceptan que les corresponde a los adultos otro lugar, desde el cual acompañar a los adolescentes, aceptando, así, que pertenecen a una generación distinta, en un momento distinto de la humanidad, con inevitable y dichosamente una nueva visión del mundo, tal vez no mejor ni peor solo distinta. Pero es a los adultos a los que le corresponde dar este giro, que no evitará como dije el enfrentamiento, pero posibilitará que este proceso aún cuando doloroso sea constructivo.

En resumen: los y las adolescentes de hoy son una generación distinta, les tocará en otro momento histórico ocupar el lugar de adultos y responder desde ese lugar, pero mientras tanto hoy, son adolescentes y les toca responder como adolescentes.

Los y las adolescentes de hoy propondrán respuestas e interrogantes desconocidas para las generaciones pasadas. Son la generación del siglo XXI, el siglo de la revolución informática, con una nueva visión estética producto de su época. No están obligados a repetir lo andado 
por las generaciones anteriores, tampoco a destruirlo sino a construir a partir de ellas, Si se les reconoce y respeta ese espacio tendrán más posibilidades de crear, pero si los adultos insisten en reprimir, vigilar y castigar, no les dejamos otra opción que la de demostrarnos con todas sus fuerzas el error, serán arrasadores en su crítica, asumirán posiciones extremas y quizá de riesgo. De mantener dicha postura el encuentro entre generaciones será siempre violento, pero no los podremos detener o eliminar, porque no se puede apagar la llama de la juventud y porque la vida se alimenta de lo nuevo.

\section{Referencias}

Bercovich, S. (1994). El Sujeto de la Adolescencia. En: Inscribir el Psicoanálisis, Año 1, N² Junio-diciembre, San José, Costa Rica.

Código de la Niñez y la Adolescencia. (1998). Ley $N^{\circ} 7739$. Aprobado por la Asamblea Legislativa de la República de Costa Rica el 3 de setiembre de 1997. Publicado en La Gaceta.

Rice, P. (1994). Adolescencia. Desarrollo, relaciones y cultura. Los Ángeles EEUU. Prentice Hall.

Rodulfo, R. (1989). El adolescente y sus trabajos. Conferencia dictada en la Escuela Argentina de Psicoterapia para graduados, Buenos Aires.

Serrat, J. (1970). Canción Señora. En: Álbum Mi Niñez. Editado en México.

Tubert, S. (1988). La muerte y lo imaginario en la Adolescencia. Madrid: Editorial Saltés. 